\title{
Association between the SIRT1 mRNA Expression and Acute Coronary Syndrome
}

\author{
Yongyan Hu${ }^{1,2}$, Laiyuan Wang ${ }^{1}$, Shufeng Chen ${ }^{1}$, Xuehui Liu ${ }^{1,2}$, Hongfan $\mathrm{Li}^{1,2}$, Xiangfeng Lu ${ }^{1}$, Xueli Yang ${ }^{1}$, \\ Jianfeng Huang ${ }^{1}$ and Dongfeng $\mathrm{Gu}^{1}$
}

\footnotetext{
${ }^{1}$ State Key Laboratory of Cardiovascular Disease, Division of Population Genetics, Fuwai Hospital \& National Center of Cardiovascular Diseases, Chinese Academy of Medical Sciences \& Peking Union Medical College, Beijing, China

${ }^{2}$ National Human Genome Center at Beijing, Beijing, China
}

\begin{abstract}
Aim: Silent mating type information regulator 2 homolog 1 (SIRT1) functions as an atheroprotective factor in vascular biology, and genetic variations in SIRT1 are associated with coronary artery calcification and type 2 diabetes in several populations. In this study, we investigated the relationship between the mRNA expression levels of the SIRT1 gene and single nucleotide polymorphisms (SNPs) in the context of acute coronary syndrome (ACS).

Methods: Whole-genome expression microarray and real-time PCR techniques were used to detect the gene expression levels, and Western blotting was performed to determine the protein expression level. The four selected SNPs were genotyped in a Taqman genotyping platform.

Results: Compared with that observed in the controls, the mRNA expression levels of the SIRT1 gene in the microarray study were significantly lower in the acute myocardial infarction (AMI), unstable angina (UA) and overall ACS patients. These results were replicated in another independent cohort with respect to the mRNA (AMI, $p<0.001$; UA, $p<0.001$; ACS, $p<0.001)$ and protein $(p<$ $0.05)$ levels. Furthermore, the relationship between the SIRT1 mRNA expression and the genotypes of four possible functional SNPs (rs12778366, rs3758391, rs2273773 and rs4746720) was tested, the results of which showed significant differences in the SIRT1 mRNA expression among the allelic genes of rs3758391 $(p<0.01)$ in the healthy participants.

Conclusions: The present results confirm that the SIRT1 gene plays a protective role against ACS and that the rs3758391 SNP affects the mRNA expression in healthy participants, providing new insight into the processes regulating the genetic control of the SIRT1 gene with respect to the pathogenesis of ACS.
\end{abstract}

J Atheroscler Thromb, 2015; 22:165-182.

Key words: SIRT1, ACS, Gene expression, SNP

\section{Introduction}

Acute coronary syndrome (ACS) is a leading cause of global morbidity and mortality, with a delete-

Address for correspondence: Laiyuan Wang, State Key Laboratory of Cardiovascular Disease, Division of Population Genetics, Fuwai Hospital \& National Center of Cardiovascular Diseases, Chinese Academy of Medical Sciences \& Peking Union Medical College, No.167 Beilishi Road, Xicheng District, Beijing, 100037, China

E-mail: wanglaiyuandw@163.com

Received: March 10, 2014

Accepted for publication: July 23, 2014 rious impact on health and substantial economic burdens due to the need for hospitalization ${ }^{1)}$. ACS includes unstable angina (UA) and acute myocardial infarction (AMI). Studies of the pathogenesis of ACS have focused primarily on atherosclerosis. The physical rupture of the atherosclerotic plaque that accumulates in patients with this condition accounts for almost all cases of acute coronary thrombi ${ }^{2}$. A large amount of laboratory research conducted within the past decade has led to many achievements describing the biological and genetic foundations of atherosclero$\operatorname{sis}^{3)}$.

Silent mating type information regulator 2 
homolog 1 (SIRT1) is the closest homolog of yeast sir2 and functions as an $\mathrm{NAD}^{+}$-dependent histone deacetylase ${ }^{4)}$, contributing to regulation of the gene expression, metabolism and aging ${ }^{5)}$. Stein S. et al. ${ }^{6}$ demonstrated that the SIRT1 protein expression in the aorta is lower in $\mathrm{ApoE}^{-/-}$mice than in wild-type mice and that SIRT1 retards the progression of atherosclerosis by preventing macrophage foam cell formation. Breitenstein A. et al. ${ }^{7)}$ also found that the SIRT1 expression in monocytes to be decreased in 16 ACS patients. SIRT1 exerts atheroprotective effects on the vascular biology by downregulating the expression of various pro-inflammatory cytokines, mediating vasodilatation via the actions of eNOS-derived nitric oxide (NO) and scavenging reactive oxygen species $(\mathrm{ROS})^{8}$.

Polymorphisms related to the SIRT1 gene have been reported to be associated with a higher incidence of type 2 diabetes ${ }^{9)}$, Alzheimer's disease ${ }^{10)}$, cholesterol metabolism, coronary artery calcification ${ }^{11)}$, greater body mass index (BMI) values, an increased risk of obesity ${ }^{12)}$, elevated amounts of body fat and blood pressure $^{13)}$, etc. In addition, the mRNA expression of the SIRT1 gene is related to single nucleotide polymorphisms (SNPs) in subjects with severe obesity ${ }^{14)}$. SNPs in the SIRT1 gene may impact the mRNA expression by altering the binding ability of transcription factors or miRNAs.

In order to determine the role of SIRT1 in ACS, we sought to compare the expression levels of SIRT1 in peripheral blood mononuclear cells (PBMCs) between ACS patients and controls using a gene expression microarray and real-time PCR replication in a large sample size. In addition, four potential functional SNPs in SRIT1 were assessed as to whether they affect the mRNA expression levels of the SIRT1 gene.

\section{Methods}

\section{Study Subjects}

A total of 58 patients with ACS were enrolled from Fuwai Hospital at the National Center of Cardiovascular Diseases (Beijing, China). All patients underwent initial diagnostic coronary angiography and were found to have either a minimum of $70 \%$ stenosis in a major epicardial artery or $50 \%$ stenosis in the left main coronary artery. The subjects were divided into two groups: (1) those with UA $(n=30$, based on the following inclusion criteria: angina pectoris with a crescendo pattern, recurrent episodes at rest/low levels of activity and/or severe new onset with ischemic changes on electrocardiography); (2) and those with AMI $(n=28$, based on the following inclusion criteria: typical chest pain lasting more than 30 minutes within 24 hours before enrollment with ischemic changes on electrocardiography and significant elevation of the creatine kinase-MB and troponin I levels). Subjects with congenital heart disease, valvular disease, cardiomyopathy, stroke, secondary hypertension, diabetes and severe renal or hepatic disease were excluded. In patients with ACS, blood samples were obtained within 48 hours after the onset of the acute event.

A group of 229 participants from the rural district of Shandong Province were enrolled in the China Cardiovascular Health Study (CCHS) project, a population-based investigation of risk factors for cardiovascular disease conducted in China since 2006 ${ }^{15}$. Individuals were excluded from the study if they had stage-two/three hypertension (systolic blood pressure $\geq 160 \mathrm{mmHg}$ and/or diastolic blood pressure $\geq 100$ $\mathrm{mmHg}$ ), a history of coronary heart disease, diabetes, stroke or renal or hepatic disease or took antihypertensive medications. One hundred and seventy-five age- and gender-matched participants were randomly selected from the above healthy participants as the control group in the SIRT1 mRNA expression replication study. Sixty-seven participants were randomly selected from the above population for the assessment of the relationship between the gene expression of SIRT1 and the inflammatory cytokine $I L-6$.

Informed consent was obtained from all participants prior to inclusion in the study. The investigation conformed to the principles outlined in the Declaration of Helsinki and was approved by the ethics review board of Fuwai Hospital (2011-341).

\section{Cell Isolation and RNA Extraction}

Peripheral blood was collected from all participants after an overnight fast, and PBMCs were isolated using Lymphocyte Separation Medium (TBD, Tianjin, China) according to the manufacturer's instructions. Total RNA was extracted from the PBMCs using TRIzol reagent (Ambion, Austin, USA) according to the manufacturer's protocol, and the RNA concentration was determined using a NanoDrop2000 spectrophotometer (Thermo Scientific, Wilmington, DE). All RNA samples were stored at $-80^{\circ} \mathrm{C}$ prior to labeling, hybridization and cDNA synthesis.

\section{Whole Genome Expression}

The quality of the RNA was determined using a 2100 Bioanylzer (Agilent Technologies, Santa Clara, CA). Samples with an RNA integrity number greater than 9.5 were examined in the subsequent expression analyses. Amplification and labeling of the RNA was performed with the Illumina TotalPrep RNA Amplifi- 
cation Kit from Life Technologies (Ambion, Austin, TX) using 150 ng of RNA per sample. Subsequently, $750 \mathrm{ng}$ of labeled cRNA was hybridized to Human HT-12 v4 Expression BeadChips (Illumina, San Diego, CA) for 18 hours and processed according to the manufacturer's instructions. The slides were then scanned using a BeadArray Reader (Illumina, San Diego, CA).

\section{Microarray Data Analysis}

The GenomeStudio Gene Expression Module was used as a tool to analyze the gene expression data obtained from the scanned microarray results generated by the Illumina BeadArray Reader. The data processing technique included a normalization method, named Average, to reduce the obscuring variation between the microarrays, which may be introduced during the process of sample preparation, manufacturing, fluorescence labeling, hybridization and/or scanning. The statistical analysis of the differential gene expression data between the case and control groups was conducted using an Illumina custom software program. The moderated $t$ statistic $p$-values derived from the Illumina custom analysis were further adjusted for multiple testing according to Benjamini and Hochberg's method ${ }^{16)}$ in order to control the false discovery rate (FDR). Differentially expressed genes were identified based on an FDR of $<10 \%$ and a $p$-value of $<0.01$ between the case and control groups.

\section{SNP Selection}

According to the findings of previous studies and functional predictions (UCSC ENCODE ${ }^{17)}$ : http:// genome.ucsc.edu/ENCODE/; FastSNP ${ }^{18)}$ : http://fastsnp.ibms.sinica.edu.tw/pages/input_CandidateGeneSearch.jsp), we selected four potential function SNPs (rs12778366, rs3758391, rs2273773 and rs4746720) in the SIRT1 gene region. On the ENCODE website, we selected layered $\mathrm{H} 3 \mathrm{~K} 27 \mathrm{Ac}$, layered $\mathrm{H} 3 \mathrm{~K} 4 \mathrm{Me} 1$, layered H3K4Me3, DNaseI Hypersensitivity Clusters and TS miRNA sites as functional criteria. Both rs3758391 and rs12778366 locate in the 5' flanking region of the SIRT1 gene. Taking into consideration that rs4746720 is located in the 3'untranslated region (UTR) of the SIRT1 gene, we used the computational prediction website miRNASNP2 ${ }^{19)}$ (July 2013 Release; http://www.bioguo.org/miRNASNP2/) to explore the miRNA targets gained or lost by the existence of rs4746720.

\section{DNA Extraction and Genotyping}

Genomic DNA was isolated from blood leukocytes according to a standard procedure using a DNA

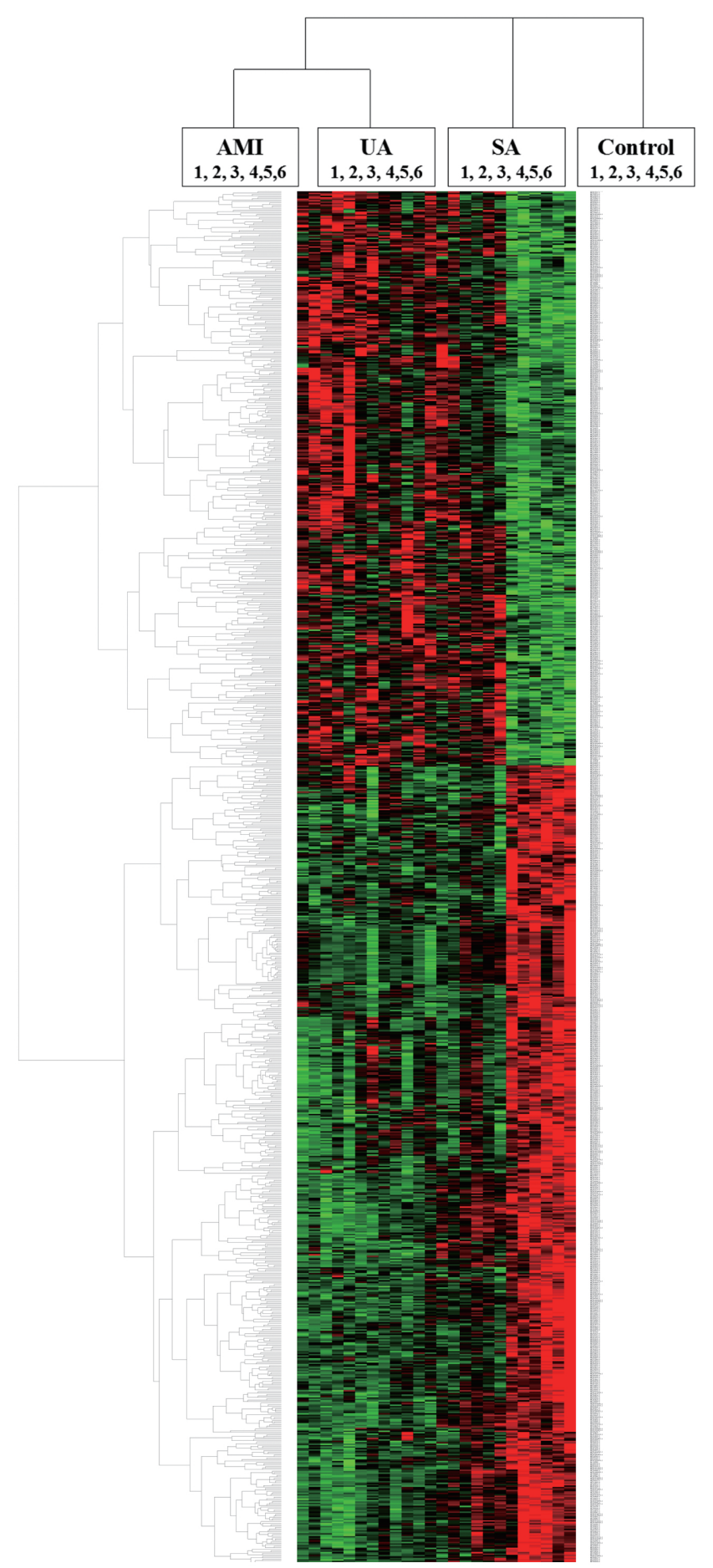

Fig. 1. Hierarchical clustering analysis of the gene expression in the peripheral blood mononuclear cells (PBMCs) of the acute myocardial infarction (AMI) patients, unstable angina (UA) patients, stable angina (SA) patients and control subjects.

Ward's Minimum Method was used. The labels AMI, UA, SA and Control represent six PBMC samples each from the AMI patients, UA patients, SA patients and control subjects, respectively. 
extraction kit (Tiangen Biotech, Beijing, China). The SNPs were genotyped using a Taqman genotyping platform (ABI 7900 HT Real-time PCR system, Applied Biosystems, Foster City, CA). The probes and primers are shown in supplementary material Supplementary Table 1 . The thermocycler parameters were as follows: $95^{\circ} \mathrm{C}$ for 10 minutes, 40 cycles of $95^{\circ} \mathrm{C}$ for 15 seconds and $60^{\circ} \mathrm{C}$ for one minute. The rate of successful genotyping was over $98 \%$, and $2 \%$ of the samples were randomly selected and genotyped in duplicate. The discrepancy between the samples was less than $1 \%$.

\section{cDNA Synthesis and Real-Time PCR Detection}

cDNA was synthesized from $1 \mu \mathrm{g}$ of RNA using reverse transcriptase with an oligo(dT) primer (TransGen Biotech, Beijing, China).

Real-time PCR for SIRT1 and IL-6 was carried out in triplicate using SYBR Green on an Applied Biosystems 7900 HT Fast Real-Time PCR System (Applied Biosystems, Foster City, USA). The relative mRNA expression levels were normalized to that of the housekeeping gene glyceraldehyde-3-phosphate dehydrogenase $(G A P D H)$ and analyzed according to the $\Delta \Delta C \mathrm{t}$ method, after which the fold changes were calculated $^{20)}$. Primers specific for the SIRT1, IL-6 and GAPDH genes are provided in supplementary material Supplementary Table 2. The amplification program consisted of one cycle at $50^{\circ} \mathrm{C}$ for two minutes then another cycle at $95^{\circ} \mathrm{C}$ for two minutes, followed by 40 cycles at $95^{\circ} \mathrm{C}$ for 15 seconds and $60^{\circ} \mathrm{C}$ for one minute. A melting curve analysis was performed after amplification in order to verify the accuracy of the amplicon.

\section{Western Blotting}

Total protein extracted from the PBMCs was separated on a $10 \%$ polyacrylamide gel and then transferred onto a nitrocellulose membrane. The membrane was subsequently incubated overnight with one of the primary antibodies against SIRT1 (sc15404, Santa Cruz Biotechnology, Santa Cruz, CA) and $\beta$-Actin (sc-47778, Santa Cruz Biotechnology, Santa Cruz, CA). Immunoblots were detected using a chemiluminescence kit (Thermo Scientific Pierce, Waltham, MA), and the autoradiographs were quantitated via densitometry (Science Imaging System, BioRad, Hercules, CA).

\section{Statistical Analysis}

The genotype distributions, Hardy-Weinberg equilibrium and differences between categorical variables were tested using the Chi-square or Fisher's exact

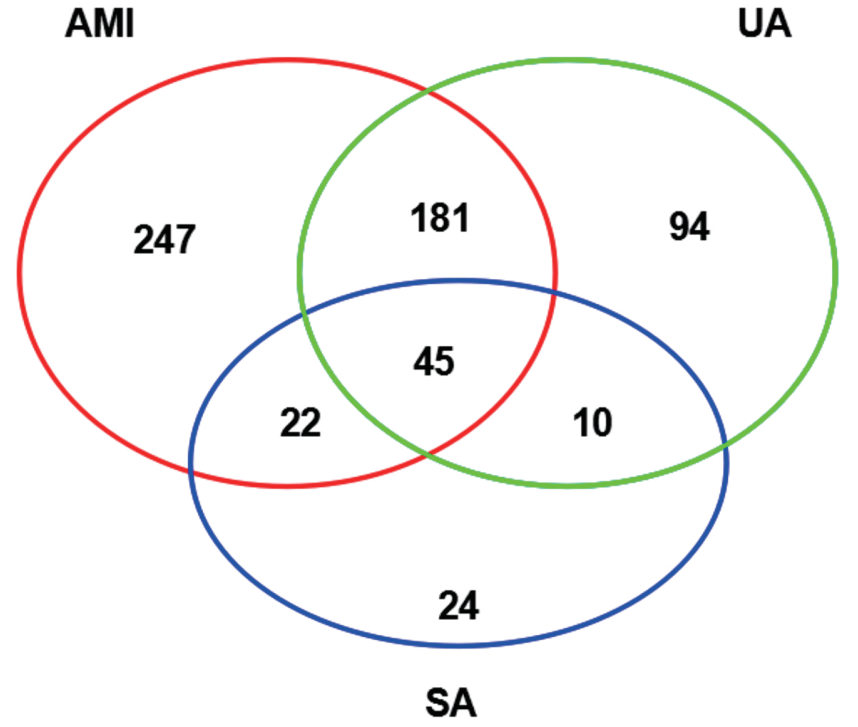

Fig. 2. Venn diagram showing the number of differentially expressed genes common to the AMI and UA patients, but not expressed in the SA patients (all relative to that observed in the control subjects). There were 181 genes unique to the acute coronary syndrome (ACS) patients.

tests. Continuous variables, patients versus controls and the different genotype groups were compared using the independent Student's $t$-test or analysis of variance (ANOVA). The HaploView 4.2 software package was used to calculate and display the linkage disequilibrium (LD) values in the gene region. The statistical analyses of the real-time PCR results and descriptive statistics of the study population were performed using the SPSS software 17.0 (SPSS Inc., Chicago, Ill) and GraphPad Prism 5.0 (GraphPad, San Diego, CA, USA) programs. All results are expressed as the mean \pm standard error of the mean (SEM), and statistical significance was defined as a $p$-value less than 0.05 .

\section{Results}

\section{Whole-Genome Expression Analysis}

A whole-genome expression analysis was performed among six AMI patients, six UA patients, six stable angina (SA) patients and six control individuals. The hierarchical clustering was performed based on the normalized signal data in order to show distinguishable gene expression profiling among the samples (Fig. 1). Genes differentially expressed in the patients relative to the controls were analyzed in an effort to distinguish genes uniquely expressed in ACS patients. A total of 181 genes common expressed in AMI and UA patients, but not in SA patients, were considered 
Table 1. Differentially expressed genes with a potential role in AMI, UA and ACS compared with that observed in the controls

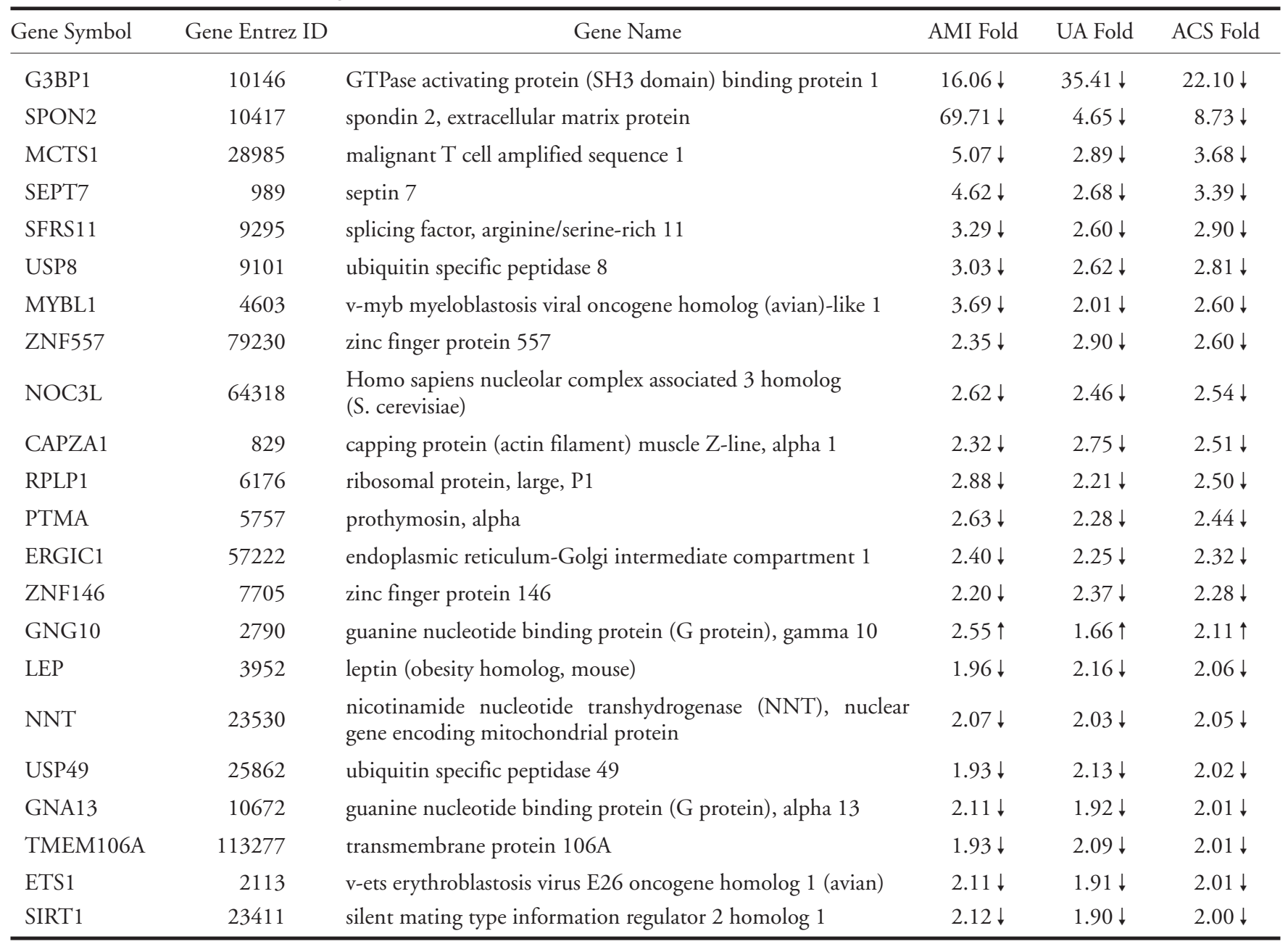

There were 75 differentially decreased expressed genes and 23 differentially increased expressed genes in the AMI, UA and ACS patients versus the controls. The complete list of all 98 genes is available in Supplementary Table 1. The subset of genes with the greatest differential expression (fold change $\geq 2$ ) is listed here. $\downarrow=$ decreased; $\uparrow=$ increased.

to be unique to ACS (shown in Fig. 2). Based on the above genes, 98 coding transcripts with well-established annotation, including 23 upregulated genes and 75 downregulated genes, were selected for the further analysis (shown in Supplementary Table 3 and Supplementary Table 4). Twenty-three genes with the greatest differential expression (fold change $>2$ ) are shown in Table 1.

We reviewed previous studies in order to examine whether the functions of the above 23 genes were related to ACS and found that the expression of SIRT1 gene had the greatest association evidence ${ }^{7,21,22)}$. Our findings showed that the expression of the SIRT1 gene was reduced considerably in the patients suffering from AMI, UA and ACS compared with that observed in the control group.

\section{Replication of the SIRT1 Expression in the Patients with ACS and the Controls}

The expression levels of the SIRT1 gene were replicated using real-time PCR. The replication cohort consisted of 58 ACS patients and 175 control subjects (Table 2). Compared with that observed in the controls $(0.90 \pm 0.72$ fold, $n=175)$, the SIRT1 mRNA expression levels were reduced in the AMI $(0.34 \pm 0.41$ fold, $p=5.37 \times 10^{-10} ; n=28$; Fig. 3A), UA $(0.43 \pm 0.37$ fold, $p=7.45 \times 10^{-7} ; n=30$; Fig. 3A) and overall ACS patients $\left(0.38 \pm 0.34\right.$ fold, $n=58 ; p=6.59 \times 10^{-12}$; Fig. 3A).

In addition, protein in the PBMCs was isolated from a subgroup of age-matched ACS patients $(n=8)$ and control subjects $(n=10)$, and levels of SIRT1 proteins were detected using a Western blot analysis 
Table 2. Demographic and clinical characteristics of the CAD and control samples

\begin{tabular}{lccc}
\hline Characteristics & Control $(n=175)$ & ACS $(n=58)$ & $p$-value* \\
\hline Gender $($ male, \%) & 70.3 & 69 & 0.85 \\
Age & $53.00 \pm 4.95$ & $56.03 \pm 7.82$ & 0.01 \\
BMI $\left(\mathrm{kg} / \mathrm{m}^{2}\right)$ & $25.60 \pm 3.23$ & $25.53 \pm 3.12$ & 0.89 \\
Smoker $(\%)$ & 23.4 & 62.1 & 0.00 \\
Drinker $(\%)$ & 45.7 & 34.5 & 0.13 \\
\hline
\end{tabular}

Continuous variables: mean \pm SD. ${ }^{*}$ Comparison of the ACS cases and controls. Independent Student's $t$-test for continuous variables, Chi-square test for categorical variables. ACS, acute coronary syndrome; BMI, body mass index.

(Fig. 3B). Consequently, the SIRT1 protein expression in the ACS patients $(0.80 \pm 0.04$ fold, $p=0.01)$ was lower than that observed in the control subjects $(1.0 \pm$ 0.06 fold). Taken together, the data in Fig. 3 suggest that patients with ACS have decreased levels of SIRT1 mRNA and proteins in their PBMCs.

\section{Relationship between the Gene Expression of SIRT1 and the Inflammatory Cytokine IL-6}

In order to determine the relationship between the SIRT1 and inflammatory cytokine $I L-6$ gene expression, the transcript levels of SIRT1 and IL-6 in PBMCs obtained from healthy individuals were measured using real-time PCR. Consequently, the level of SIRT1 negatively correlated with the inflammatory cytokine $I L-6$ gene expression $(r=-0.45, p=0.0002, n=67)($ Fig. 4).

\section{Functional SNP Prediction}

In order to further explore the mechanisms underlying the regulation of the SIRT1 gene in the setting of CAD with respect to genetic variants, we applied a bioinformatics tools to predict potential functional SNPs and subsequently evaluated the associations between the genotypes and the SIRT1 mRNA expression. A search for potential functional features of the four SNPs on the ENCODE website showed that rs12778366 and rs3758391 were located in the enriched zone of $\mathrm{H} 3 \mathrm{~K} 4 \mathrm{Me} 1$ markers, which are often found near regulatory elements (Fig. 5). In addition, rs4746720 was found to be located in an miRNA regulatory site on both the ENCODE and miRNASNP2 websites. The mutation of rs4746720 from $\mathrm{T}$ to $\mathrm{C}$ creates a binding site for hsa-miR-2115-3p (Supplementary Fig. 1). Rs2273773, which has been reported to be associated with body fat and blood pressure, is a synonymous variant in exon 5 of the SIRT1 gene ${ }^{13)}$. Hence, the above four SNPs were selected to examine whether their genotypes affect the mRNA expression of the SIRT1 gene.
Association between the mRNA Expression Level of SIRT1 and the Presence of the Four SNPs in the General Subjects

Genotyping of the four SNPs and real-time PCR detection of the SIRT1 gene were implemented in the PBMC samples obtained from 58 ACS patients and 229 healthy participants. Among the healthy participants, the genotype frequencies of rs12778366, rs3758391, rs2273773 and rs4746720 were in compliance with the Hardy-Weinberg equilibrium ( $p=$ $0.22, p=0.84, p=0.91$ and $p=0.66$, respectively). We then analyzed the association between genotyping and the gene expression. However, there were no significant differences in the gene expression among the ACS patients with different genotypes (Supplementary Fig. 2).

We subsequently analyzed the allelic expression of rs12778366, rs3758391, rs2273773 and rs4746720 among the healthy participants and found notable differences for rs3758391. For example, the mRNA expression levels of SIRT1 in rs3758391 were significantly higher in 175 individuals with the TT genotype $(0.92 \pm 0.08$ fold $)$ than in 54 individuals with the TC and CC genotypes $(0.63 \pm 0.07$ fold, $p=0.006$; Fig. 6A; only four CC homozygotes for rs3758391, thus TC and CC were combined). The characteristics and rs3758391 genotype distributions of the study participants are shown in Supplementary Table 5.

In contrast, the rs 12778366 genotype was not associated with the SIRT1 mRNA expression (TT, $0.85 \pm 0.07$ fold, $n=178$; TC + CC, $0.88 \pm 0.14$ fold, $n=48 ; p=0.80$; Fig. 6B; only five CC homozygotes for rs3758391, thus TC and CC were combined). The characteristics and rs 12778366 genotype distributions of the study participants are shown in Supplementary Table 6. The SIRT1 expression levels did not differ among the genotypes of rs2273773 (TT, $0.81 \pm 0.07$ fold, $n=121$; TC, $0.96 \pm 0.12$ fold, $n=89$; CC, $0.62 \pm$ 0.10 fold, $n=17 ; p=0.31$; Fig. 6C). The characteristics and rs2273773 genotype distributions of the study participants are shown in Supplementary Table 
A

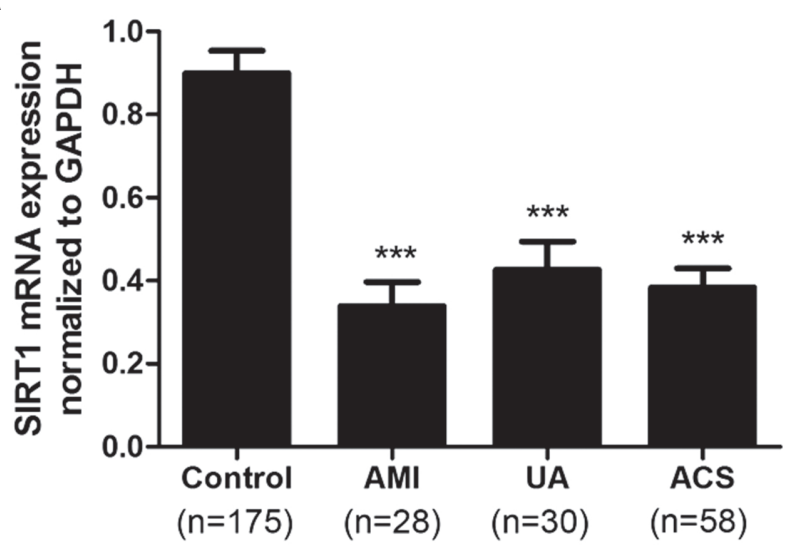

B
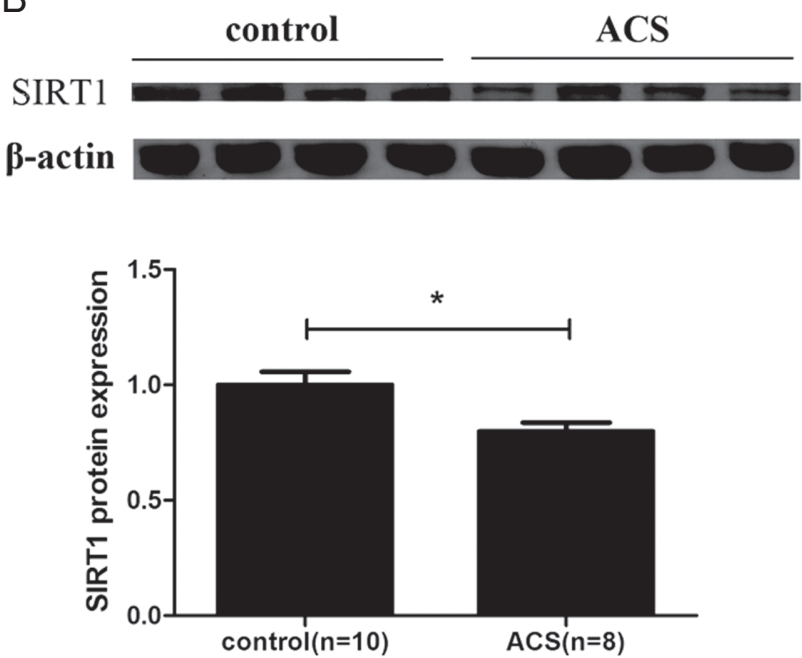

Fig. 3. Decreased levels of SIRT1 mRNA and proteins in the peripheral blood mononuclear cells (PBMCs) obtained from the patients with acute coronary syndrome (ACS). A. Decreased levels of SIRT1 mRNA in the PBMCs derived from the patients with AMI, UA and ACS (control group, $0.90 \pm 0.72$ fold, $n=175$; AMI group, $0.34 \pm 0.41$ fold, $n=28$; UA group, $0.43 \pm 0.37$ fold, $n=30$; ACS group, $n=58$ ).

B. The levels of SIRT1 proteins were determined using a Western blot analysis. Top, representative Western blots; bottom, the results were quantified from the pixel values in grey scale (control group, $1.0 \pm 0.06$ fold, $n=10$; ACS group, $0.80 \pm 0.04$ fold, $n=8$ ).

The data are presented as the mean \pm SEM. ${ }^{*} p<0.05$; ${ }^{* *} p<0.01,{ }^{* * *} p<0.001$, compared with the control. Independent Student's $t$-test

7. Meanwhile, the genotypes of rs4746720 were not related to the SIRT1 mRNA expression (TT, $0.64 \pm$ 0.07 fold, $n=64$; TC, $1.00 \pm 0.11$ fold, $n=111$; CC, $0.79 \pm 0.10$ fold, $n=54 ; p=0.06$; Fig. $6 \mathrm{D}$ ). The char-

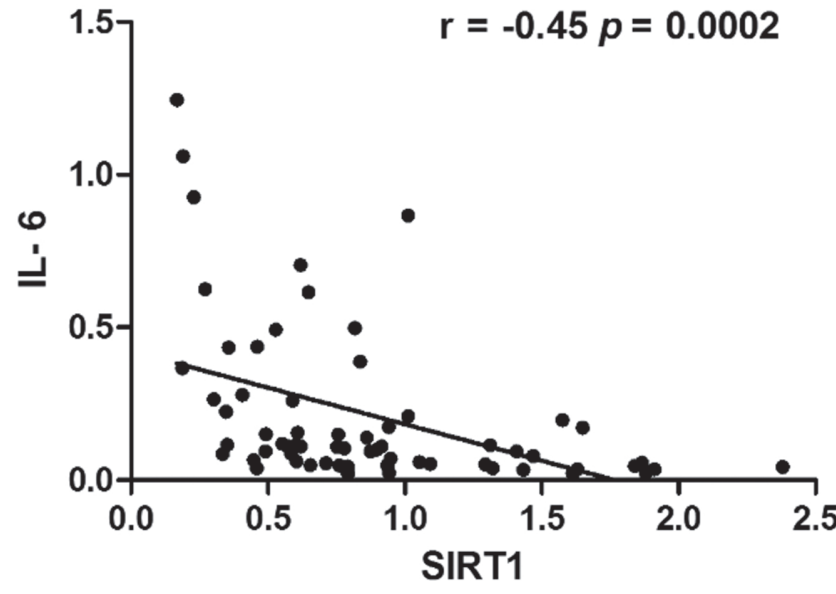

Fig. 4. Negative correlation between the SIRT1 mRNA level and the inflammatory cytokine IL- 6 gene expression. $r=-0.45, p=0.0002$.

acteristics and rs4746720 genotype distributions of the study participants are shown in Supplementary Table 8.

\section{Discussion}

In the present study, the SIRT1 mRNA expression was found to be reduced in the PBMCs obtained from the ACS patients. Furthermore, we found that rs3758391 was significantly associated with the expression of the SIRT1 gene in the healthy participants.

Our case-control study using gene expression microarrays and real-time PCR replication demonstrated that the SIRT1 mRNA expression level is associated with ACS, with a decreased expression in the PBMCs of the ACS patients, while earlier studies of the SIRT1 gene expression reported a reduced expression of the SIRT1 gene in monocytes derived from ACS patients ${ }^{7}$. PBMCs and monocytes are both cells related to the pathogenesis of ACS. Furthermore, it has been reported that $p 66 S h c$ is negatively regulated by $S I R T 1^{21)}$, while another study ${ }^{22)}$ showed that the expression of p66Shc is increased in PBMCs in ACS patients. It has also been reported that the blood levels of SIRT1 proteins are increased in patients suffering from cardiovascular disease (CVD) compared to that noted in healthy subjects ${ }^{23)}$. These observations conflict with the results of our studies of ACS and another study of coronary artery disease ${ }^{7)}$. In addition, we compared the mRNA expression levels of the SIRT1 gene in the AMI and UA patients and found that the SIRT1 expression levels were lower in the AMI patients than in the UA patients. This finding suggests that the SIRT1 expression is more significantly related 


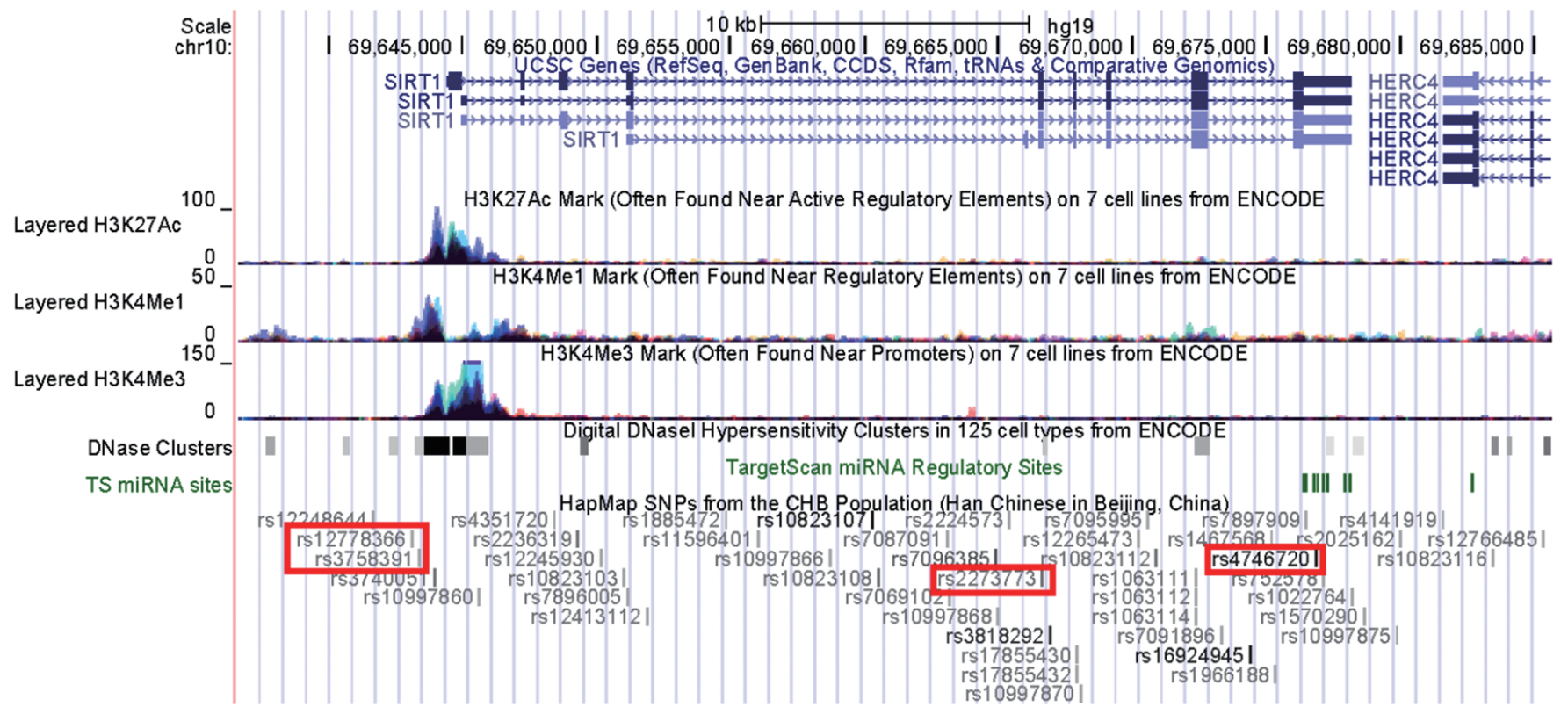

Fig. 5. ENCODE functional annotation of the SIRT1 gene with rs12778366, rs3758391, rs2273773 and rs4746720.

$\mathrm{H} 3 \mathrm{~K} 27 \mathrm{Ac}$, acetylation of lysine 27 of the H3 histone protein; H3K4me1, mono-methylation of lysine 4 of the H3 histone protein; $\mathrm{H} 3 \mathrm{~K} 4 \mathrm{Me} 3$, tri-methylation of lysine 4 of the $\mathrm{H} 3$ histone protein.

to AMI than to UA, although no studies have been conducted to explain this result.

ACS is the result of the physical rupture of atherosclerotic plaque, and inflammation plays a major role in all stages of atherogenesis ${ }^{2)}$. In addition, SIRT1 exerts anti-inflammatory effects in the setting of atherosclerosis $^{24)}$, and the level of SIRT1 negatively correlated with the gene expression of the inflammatory cytokine IL- 6 in our study. Moreover, it has been reported that ACS patients have significantly elevated levels of the inflammatory cytokine $I L-\sigma^{25)}$ and that the knockdown of SIRT1 results in an increased IL- 6 expression ${ }^{26}$. These findings indicate that inflammation is a contributing factor affecting the downregulation of the SIRT1 gene expression.

Another contributing factor may be the effects of the non-coding genetic variant of the SIRT1 gene. Increasing evidence suggests that non-coding genetic variants impact the gene expression by binding to different transcription factors ${ }^{27)}$. In the present study, we observed a strong relationship between rs3758391 and the SIRT1 mRNA expression in healthy individuals, and rs3758391 was not in LD with the other three SNPs (Supplementary Table 9). It has also been reported that rs3758391 is associated with the calorie restriction-stimulated SIRT1 expression and may affect the SIRT1 mRNA expression via the loss of binding sites of the transcription factor $\mathrm{p} 53$. The $\mathrm{C}$ allele of rs3758391 disrupts the mirror-image symme- try of the p53 consensus binding sequence ${ }^{28)}$. Therefore, the change from $\mathrm{T}$ to $\mathrm{C}$ in a conserved region of the p53-binding element decreases binding to $\mathrm{p} 53$ in vitro. Furthermore, p53 has been found to induce myocyte apoptosis via activation of the renin-angiotensin system ${ }^{29)}$. The high SIRT1 expression of the T allele of rs3758391 may be the result of a protective negative feedback loop with respect to the detrimental effects of p53. To our knowledge, the present study is the first to confirm this finding in the general population. Finally, SIRT1 has been found to deacetylate p55 and regulate the functions of p53 in cells ${ }^{30)}$. That is to say, rs3758391 regulates the SIRT1 expression by changing the $\mathrm{p} 53$ binding site. Therefore, inflammation and SIRT1-related genetic variants may be contributing factors affecting the downregulation of the SIRT1 gene expression.

We also analyzed the association between the presence of the four functional SNPs and the SIRT1 mRNA expression in the ACS patients; however, we did not find any significant differences among the different genotypes. The primary explanation for this finding is the use of medications prior to blood sampling in the ACS patients, which may have affected the SIRT1 gene expression.

Concerning rs2273773, we found no significant associations with the SIRT1 mRNA expression, although the discrepant data for the alleles with respect to fasting glucose, BMI and diastolic blood pressure 
A

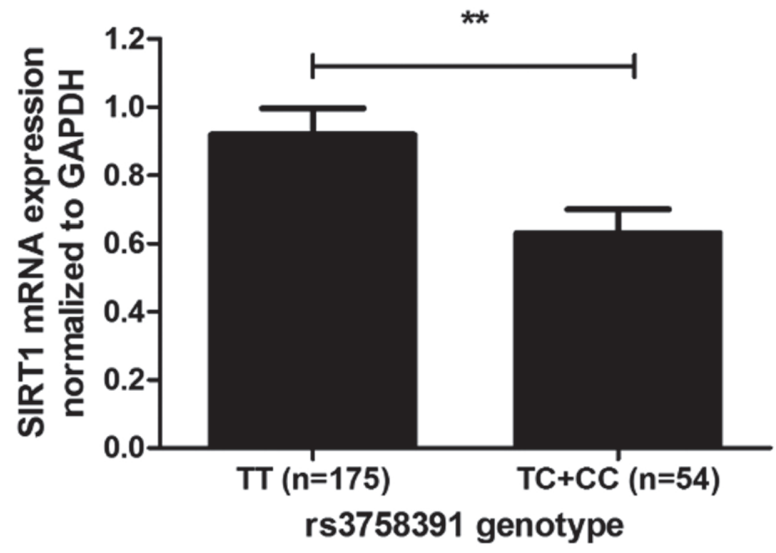

C

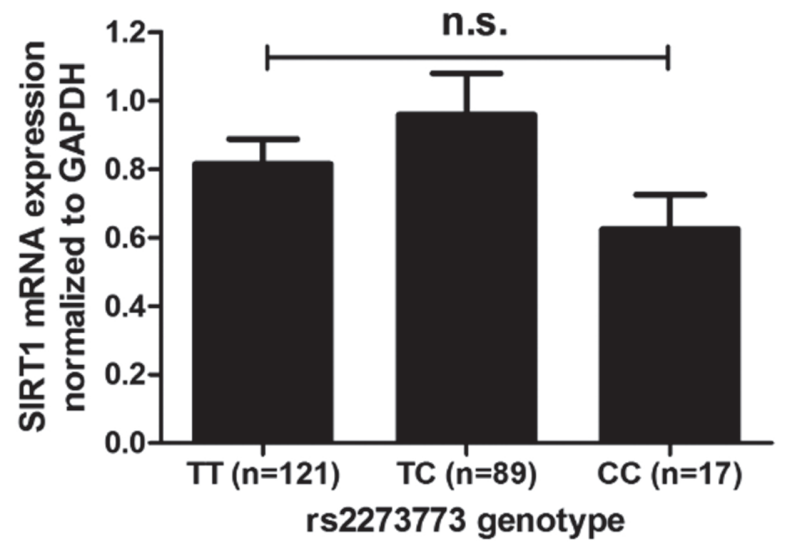

B

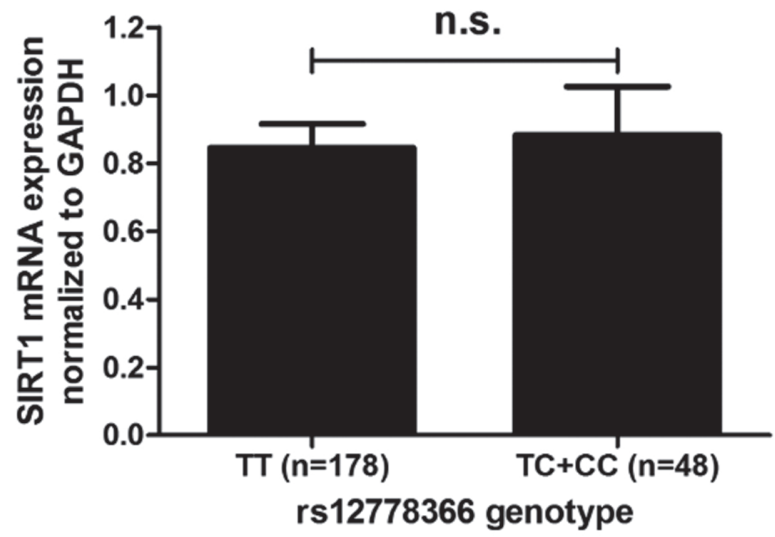

D

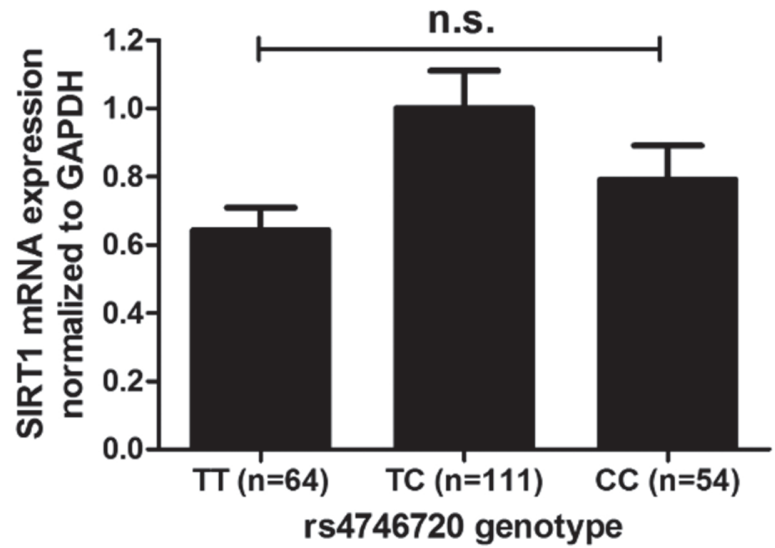

Fig. 6. Relationship between the SIRT1 mRNA expression levels in the healthy subjects and the genotypes of rs 3758391 , rs 4746720 , rs12778366 and rs2273773.

A. Expression levels of SIRT1 mRNA according to the different genotypes of rs3758391 (TT, $0.92 \pm 0.08$ fold, $n=175$; TC + CC, $0.63 \pm 0.07$ fold, $n=54$; only four CC homozygotes for rs3758391, thus TC and CC were combined. Independent Student's $t$-test).

B. Expression levels of SIRT1 mRNA according to the different genotypes of rs12778366 (TT, $0.85 \pm 0.07$ fold, $n=178$; TC + CC, $0.88 \pm 0.14$ fold, $n=48$; only five CC homozygotes for rs 12778366 , thus TC and CC were combined. Independent Student's $t$-test).

C. Expression levels of SIRT1 mRNA according to the different genotypes of rs 2273773 (TT, $0.81 \pm 0.07$ fold, $n=121$; TC, $0.96 \pm 0.12$ fold, $n=89$; CC, $0.62 \pm 0.10$ fold, $n=17$. Analysis of variance).

D. Expression levels of SIRT1 mRNA according to the different genotypes of rs4746720 (TT, $0.64 \pm 0.07$ fold, $n=64$; TC, $1.00 \pm 0.11$ fold, $n=111$; CC, $0.79 \pm 0.10$ fold, $n=54$. Analysis of variance).

The data are presented as the mean \pm SEM. ${ }^{* *} p<0.01$; n.s., non significant.

(Supplementary Table 7) were in accordance with the findings of a Japanese health checkup examinee study ${ }^{13)}$. Although rs2273773 is a synonymous mutation, it may act via noncoding functions pre-transcriptionally at the DNA level or post-transcriptionally at the RNA level ${ }^{27)}$. SIRT1 participates in fat mobilization via the actions of peroxisome proliferator-acti- vated receptor- $y(\operatorname{PPAR} \gamma)^{31)}$, which inhibits the SIRT1 expression at the transcriptional level. Rs2273773 may therefore affect BMI and blood pressure via PPAR $y$. Our results are the first to indicate this phenomenon in a Chinese Han population, although further confirmation is required in other populations with larger sample sizes. 
The limitation of our study is that the actions of PBMCs only partly reflect the biological events occurring in the vessel wall during the inflammatory response. However, endothelial and/or smooth muscle cell samples, which may better represent the condition of the coronary vessels, were inaccessible in this study due to the large sample size. In addition, the complexity of our population study means that the results must be verified in a larger sample.

\section{Conclusion}

The present findings further confirm that the SIRT1 mRNA expression is reduced in ACS patients and that the gene SIRT1 therefore confers a positive effect against the development of ACS. In addition, the detection of rs3758391 may be used in SIRT1related disease prediction as a biomarker. The fact that the change in SIRT1 mRNA is associated with the presence of nearby SNPs provides insight into the mechanisms by which common noncoding genetic variants affect the target gene expression via the actions of transcription factors. Hence, a more thorough functional analysis is warranted to verify the role of these variants in the pathogenesis of ACS.

\section{Acknowledgements}

This work was supported by grants from the National Basic Research Program of China (973 Plan) (2011CB503901) and the National Science Foundation of China (81273153 and 81270334).

\section{Conflicts of Interest}

None declared.

\section{References}

1) Delgado GA, Abbott JD: Acute Coronary Syndromes. In: Evidence-Based Cardiology Consult. Springer, 2014; 15-36

2) Libby P, Theroux P: Pathophysiology of coronary artery disease. Circulation, 2005; 111: 3481-3488

3) Libby P, Ridker PM, Hansson GK: Progress and challenges in translating the biology of atherosclerosis. Nature, 2011; 473: 317-325

4) Gasser SM, Cockell MM: The molecular biology of the SIR proteins. Gene, 2001; 279: 1-16

5) Pillarisetti S: A review of Sirt1 and Sirt1 modulators in cardiovascular and metabolic diseases. Recent Pat Cardiovasc Drug Discov, 2008; 3: 156-164

6) Stein S, Lohmann C, Schäfer N, Hofmann J, Rohrer L, Besler C, Rothgiesser KM, Becher B, Hottiger MO, Borén J: SIRT1 decreases Lox-1-mediated foam cell for- mation in atherogenesis. Eur Heart J, 2010; 31: 2301 2309

7) Breitenstein A, Wyss CA, Spescha RD, Franzeck FC, Hof D, Riwanto M, Hasun M, Akhmedov A, von Eckardstein A, Maier W: Peripheral Blood Monocyte Sirt1 Expression Is Reduced in Patients with Coronary Artery Disease. PloS One, 2013; 8: e53106

8) Stein S, Matter CM: Protective roles of SIRT1 in atherosclerosis. Cell Cycle, 2011, 10: 640-647

9) Zillikens MC, van Meurs JB, Sijbrands EJ, Rivadeneira F, Dehghan A, van Leeuwen JP, Hofman A, van Duijn CM, Witteman J, Uitterlinden AG: SIRT1 genetic variation and mortality in type 2 diabetes: interaction with smoking and dietary niacin. Free Radical Biol Med, 2009; 46: 836-841

10) Kuningas M, Putters M, Westendorp RG, Slagboom PE, van Heemst D: SIRT1 gene, age-related diseases, and mortality: the Leiden 85-plus study. J Gerontol A Biol Sci Med Sci, 2007; 62: 960-965

11) Shimoyama Y, Mitsuda Y, Tsuruta Y, Suzuki K, Hamajima N, Niwa T: SIRTUIN 1 Gene Polymorphisms are Associated With Cholesterol Metabolism and Coronary Artery Calcification in Japanese Hemodialysis Patients. J Ren Nutr, 2012; 22: 114-119

12) Zillikens MC, van Meurs JB, Rivadeneira F, Amin N, Hofman A, Oostra BA, Sijbrands EJ, Witteman JC, Pols HA, van Duijn CM: SIRT1 genetic variation is related to BMI and risk of obesity. Diabetes, 2009; 58: 2828-2834

13) Shimoyama Y, Suzuki K, Hamajima N, Niwa T: Sirtuin 1 gene polymorphisms are associated with body fat and blood pressure in Japanese. Transl Res, 2011; 157: 339347

14) Clark SJ, Falchi M, Olsson B, Jacobson P, Cauchi S, Balkau B, Marre M, Lantieri O, Andersson JC, Jernås M: Association of sirtuin 1 (SIRT1) gene SNPs and transcript expression levels with severe obesity. Obesity, 2012; 20: 178-185

15) Lu X, Wang L, Chen S, He L, Yang X, Shi Y, Cheng J, Zhang L, Gu CC, Huang J, Wu T, Ma Y, Li J, Cao J, Chen J, Ge D, Fan Z, Li Y, Zhao L, Li H, Zhou X, Chen L, Liu D, Duan X, Hao Y, Lu F, Liu Z, Yao C, Shen C, Pu X, Yu L, Fang X, Xu L, Mu J, Wu X, Zheng R, Wu N, Zhao Q, Liu X, Wang M, Yu D, Hu D, Ji X, Guo D, Sun D, Wang Q, Yang Y, Liu F, Mao Q, Liang X, Ji J, Chen P, Mo X, Li D, Chai G, Tang Y, Li X, Du Z, Dou C, Yang Z, Meng Q, Wang D, Wang R, Yang J, Schunkert H, Samani NJ, Kathiresan S, Reilly MP, Erdmann J, Peng X, Chen R, Qiang B, Gu D: Genome-wide association study in Han Chinese identifies four new susceptibility loci for coronary artery disease. Nat Genet, 2012; 44: 890-894

16) Benjamini $Y$, Hochberg $Y$ : Controlling the false discovery rate: a practical and powerful approach to multiple testing. J R Stat Soc Series B Stat Methodol, 1995; 289-300

17) Feingold E, Good P, Guyer M, Kamholz S, Liefer L, Wetterstrand K, Collins F, Gingeras T, Kampa D, Sekinger E: The ENCODE (ENCyclopedia of DNA elements) project. Science, 2004; 306: 636-640

18) Yuan H-Y, Chiou J-J, Tseng W-H, Liu C-H, Liu C-K, Lin Y-J, Wang H-H, Yao A, Chen Y-T, Hsu C-N: FASTSNP: an always up-to-date and extendable service for 
SNP function analysis and prioritization. Nucleic Acids Res, 2006; 34: W635-W641

19) Gong J, Tong Y, Zhang HM, Wang K, Hu T, Shan G, Sun J, Guo AY: Genome-wide identification of SNPs in microRNA genes and the SNP effects on microRNA target binding and biogenesis. Hum Mutat, 2012; 33: 254263

20) Livak KJ, Schmittgen TD: Analysis of Relative Gene Expression Data Using Real-Time Quantitative PCR and the $2^{-\Delta \Delta \mathrm{CT}}$ Method. Methods, 2001; 25: 402-408

21) Chen H-Z, Wan Y-Z, Liu D-P: Cross-talk between SIRT1 and p66Shc in vascular diseases. Trends Cardiovasc Med, 2013; 23: 237-241

22) Franzeck FC, Hof D, Spescha RD, Hasun M, Akhmedov A, Steffel J, Shi Y, Cosentino F, Tanner FC, von Eckardstein A: Expression of the aging gene p66Shc is increased in peripheral blood monocytes of patients with acute coronary syndrome but not with stable coronary artery disease. Atherosclerosis, 2012; 220: 282-286

23) Kilic U, Gok O, Bacaksiz A, Izmirli M, Elibol-Can B, Uysal O: SIRT1 gene polymorphisms affect the protein expression in cardiovascular diseases. PLoS One, 2014; 9: e90428

24) Winnik S, Stein S, Matter CM: SIRT1 - an anti-inflammatory pathway at the crossroads between metabolic disease and atherosclerosis. Curr Vasc Pharmacol, 2012; 10 : 693-696

25) Oudi ME, Aouni Z, Mazigh C, Khochkar R, Gazoueni E,
Haouela H, Machghoul S: Homocysteine and markers of inflammation in acute coronary syndrome. Exp Clin Cardiol, 2010; 15: e25-28

26) Zhu X, Liu Q, Wang M, Liang M, Yang X, Xu X, Zou H, Qiu J: Activation of Sirt1 by resveratrol inhibits TNFalpha induced inflammation in fibroblasts. PLoS One, 2011; 6: e27081

27) Ward LD, Kellis M: Interpreting noncoding genetic variation in complex traits and human disease. Nat Biotechnol, 2012; 30: 1095-1106

28) Naqvi A, Hoffman TA, DeRicco J, Kumar A, Kim C-S, Jung S-B, Yamamori T, Kim Y-R, Mehdi F, Kumar S: A single-nucleotide variation in a p53-binding site affects nutrient-sensitive human SIRT1 expression. Hum Mol Genet, 2010; 19: 4123-4133

29) Pierzchalski P, Reiss K, Cheng W, Cirielli C, Kajstura J, Nitahara JA, Rizk M, Capogrossi MC, Anversa P: p53 induces myocyte apoptosis via the activation of the reninangiotensin system. Exp Cell Res, 1997; 234: 57-65

30) Luo J, Nikolaev AY, Imai S, Chen D, Su F, Shiloh A, Guarente L, Gu W: Negative control of p53 by Sir2alpha promotes cell survival under stress. Cell, 2001; 107: 137 148

31) Picard F, Kurtev M, Chung N, Topark-Ngarm A, Senawong T, De Oliveira RM, Leid M, McBurney MW, Guarente L: Sirt1 promotes fat mobilization in white adipocytes by repressing PPAR- $\gamma$. Nature, 2004; 429: 771-776 
Supplementary Table 1.

Primer and probe sequences of variants at the SIRT1 locus

\begin{tabular}{ll}
\hline \multicolumn{1}{c}{ SNP probes } & \multicolumn{1}{c}{ Sequences } \\
\hline rs3758391-F & 5'- TGCCATAACAAACACTGGCTCTA -3' \\
rs3758391-R & 5'- GCACACTGTGACTCCATATCTAATCTTA -3' \\
rs3758391-P-T & 5'- FAM-ATCTACCATGGGTTATAT-MGB -3' \\
rs3758391-P-C & 5'- HEX-ATCTACCACGGGTTAT-MGB -3' \\
rs12778366-F & 5'- GCTTCTAGGACTGGAGATGATTACTTTC -3' \\
rs12778366-R & 5'- TCCTATCTACATCCAAAAGTCTTATTTCA -3' \\
rs12778366-P-T & 5'- FAM-AAATGAA TAGTGGTGACCAG-MGB -3' \\
rs12778366-P-C & 5'- HEX-AAATGAACAGTGGTGACC-MGB -3' \\
rs4746720-F & 5'- CACTTTTCTTTGTAACATTGAATGGTTT -3' \\
rs4746720-R & 5'- TGCCAGTGTTTAAAAATAATTGTGTT -3' \\
rs4746720-P-C & 5'- FAM-CGCTAAACTTCTGATTC-MGB -3' \\
rs4746720-P-T & 5'- HEX-CGCTAAACTT TTGATTC-MGB -3' \\
rs2273773-F & 5'- TCCAGCCATCTCTCTGTCACAA -3' \\
rs2273773-R & 5'- GCGTGTCTATGTTCTGGGTATAGTTG -3' \\
rs2273773-P-T & 5'- FAM-TTCATAGCCTTGTCAGAT-MGB -3' \\
rs2273773-P-C & 5'- HEX-TTCATAGCCCTGTCAGA-MGB -3' \\
\hline
\end{tabular}

F, forward; R, reverse; P, probe; Bold and italic letters denote SNP alleles.

\section{Supplementary Table 2.}

Primers used to evaluate the expression levels of SIRT1 and IL-6 on real-time PCR

\begin{tabular}{llc}
\hline \multicolumn{1}{c}{ Gene } & \multicolumn{1}{c}{ Sequences } & Amplicon size (bp) \\
\hline SIRT1-F & 5'- ATGCAAGCTCTAGTGACTGGACT -3' & 134 \\
SIRT1-R & 5'- CTCAGGTGGAGGTATTGTTTCC -3' & \\
IL-6-F & 5'- GATGGCTGAAAAAGATGGATGC -3' & 230 \\
IL-6-R & 5'- TGGTTGGGTCAGGGGTGGTT -3' & 6 \\
GAPDH-F & 5'- AGCCACATCGCTCAGACAC -3' & \\
GAPDH-R & 5'- GCCCAATACGACCAAATCC -3' & \\
\hline
\end{tabular}

F, forward; R, reverse. 
Supplementary Table 3.

Differentially upregulated genes with a potential role in AMI, UA and ACS compared with that observed in the controls

\begin{tabular}{lrlccc}
\hline Gene Symbol & Gene Entrez ID & \multicolumn{1}{c}{ Gene Name } & AMI Fold & UA Fold & ACS Fold \\
\hline GNG10 & 2790 & guanine nucleotide binding protein (G protein), gamma 10 & 2.55 & 1.66 & 2.11 \\
GNAQ & 2776 & guanine nucleotide binding protein (G protein), q polypeptide & 1.83 & 1.91 & 1.87 \\
LOC401357 & 401357 & hypothetical LOC401357 & 1.89 & 1.73 & 1.81 \\
RRAGD & 58528 & Ras-related GTP binding D & 1.86 & 1.70 & 1.78 \\
ZNF394 & 84124 & zinc finger protein 394 & 1.63 & 1.76 & 1.70 \\
IMPDH1 & 3614 & IMP (inosine monophosphate) dehydrogenase 1 & 1.76 & 1.63 & 1.69 \\
MAP3K7IP1 & 10454 & mitogen-activated protein kinase kinase kinase 7 interacting protein 1 & 1.74 & 1.60 & 1.67 \\
EVI2B & 2124 & ecotropic viral integration site 2B & 1.71 & 1.61 & 1.66 \\
C10orf32 & 119032 & chromosome 10 open reading frame 32 & 1.57 & 1.69 & 1.63 \\
PGAM4 & 441531 & phosphoglycerate mutase family member 4 & 1.67 & 1.58 & 1.63 \\
GRB2 & 2885 & growth factor receptor-bound protein 2 & 1.72 & 1.49 & 1.60 \\
MOBKL3 & 25843 & MOB1, Mps One Binder kinase activator-like 3 (yeast) & 1.62 & 1.55 & 1.58 \\
CMTM7 & 112616 & CKLF-like MARVEL transmembrane domain containing 7 & 1.54 & 1.57 & 1.55 \\
PTPRC & 5788 & protein tyrosine phosphatase, receptor type, C & 1.60 & 1.46 & 1.53 \\
EIF3I & 8668 & eukaryotic translation initiation factor 3, subunit I & 1.53 & 1.52 & 1.52 \\
SPG21 & 51324 & spastic paraplegia 21 (autosomal recessive, Mast syndrome) & 1.56 & 1.47 & 1.52 \\
TIMP1 & 7076 & TIMP metallopeptidase inhibitor 1 & 1.67 & 1.35 & 1.51 \\
TRAK1 & 22906 & trafficking protein, kinesin binding 1 & 1.45 & 1.50 & 1.48 \\
CARM1 & 10498 & coactivator-associated arginine methyltransferase 1 & 1.40 & 1.37 & 1.39 \\
LOC200030 & 200030 & neuroblastoma breakpoint family, member 11-like & 1.39 & 1.38 & 1.38 \\
UBE2D3 & 7323 & ubiquitin-conjugating enzyme E2D 3 (UBC4/5 homolog, yeast) & 1.40 & 1.35 & 1.38 \\
H3F3A & 3020 & H3 histone, family 3A & 1.33 & 1.32 & 1.32 \\
PABPC1 & 26986 & Homo sapiens poly(A) binding protein, cytoplasmic 1 & 1.32 & 1.32 & 1.32 \\
\hline & & & &
\end{tabular}

AMI, acute myocardial infarction; UA, unstable angina; ACS, acute coronary syndrome. 
Supplementary Table 4.

Differentially downregulated genes with a potential role in AMI, UA and ACS compared with that observed in the controls

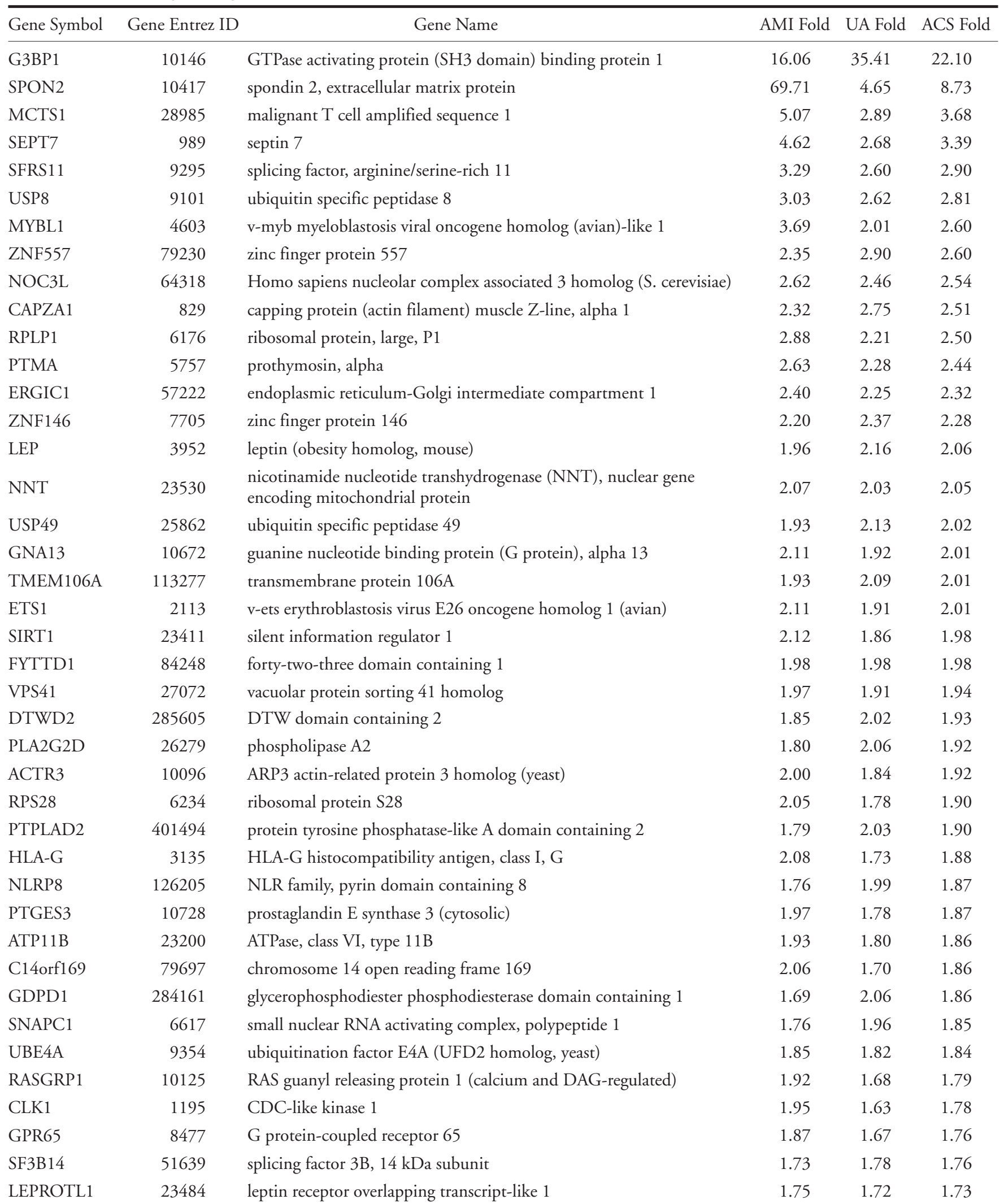


(Cont Supplementary Table 4)

\begin{tabular}{|c|c|c|c|c|c|}
\hline Gene Symbol & Gene Entrez ID & Gene Name & AMI Fold & UA Fold & ACS Fold \\
\hline RPL23 & 9349 & ribosomal protein L23 & 1.85 & 1.62 & 1.73 \\
\hline IPP & 3652 & intracisternal A particle-promoted polypeptide & 1.64 & 1.83 & 1.73 \\
\hline FASTK & 10922 & Fas-activated serine/threonine kinase & 1.69 & 1.78 & 1.73 \\
\hline DENR & 8562 & density-regulated protein & 1.71 & 1.74 & 1.73 \\
\hline MTMR6 & 9107 & myotubularin related protein 6 & 1.83 & 1.61 & 1.71 \\
\hline UBLCP1 & 134510 & ubiquitin-like domain containing CTD phosphatase 1 & 1.72 & 1.68 & 1.70 \\
\hline ZNF223 & 7766 & zinc finger protein 223 & 1.61 & 1.77 & 1.68 \\
\hline SPTLC1 & 10558 & serine palmitoyltransferase, long chain base subunit 1 & 1.58 & 1.79 & 1.68 \\
\hline KIAA1370 & 56204 & KIAA1370 & 1.80 & 1.56 & 1.67 \\
\hline RPL9 & 6133 & ribosomal protein L9 & 1.67 & 1.65 & 1.66 \\
\hline AFTPH & 54812 & aftiphilin & 1.61 & 1.70 & 1.66 \\
\hline EXOSC10 & 5394 & exosome component 10 & 1.74 & 1.57 & 1.65 \\
\hline WSB1 & 26118 & WD repeat and SOCS box-containing 1 & 1.50 & 1.82 & 1.64 \\
\hline MST4 & 51765 & serine/threonine protein kinase MST4 & 1.79 & 1.52 & 1.64 \\
\hline YIPF3 & 25844 & Yip1 domain family, member 3 & 1.55 & 1.74 & 1.64 \\
\hline BIRC3 & 330 & baculoviral IAP repeat-containing 3 & 1.53 & 1.74 & 1.62 \\
\hline EIF2AK4 & 440275 & eukaryotic translation initiation factor 2 alpha kinase 4 & 1.49 & 1.79 & 1.62 \\
\hline SKA2 & 348235 & spindle and kinetochore associated complex subunit 2 & 1.69 & 1.56 & 1.62 \\
\hline CUL4A & 8451 & cullin $4 \mathrm{~A}$ & 1.72 & 1.53 & 1.62 \\
\hline ZNF669 & 79862 & zinc finger protein 669 & 1.51 & 1.72 & 1.61 \\
\hline IBTK & 25998 & inhibitor of Bruton agammaglobulinemia tyrosine kinase & 1.54 & 1.67 & 1.61 \\
\hline DDX17 & 10521 & DEAD (Asp-Glu-Ala-Asp) box polypeptide 17 & 1.59 & 1.56 & 1.57 \\
\hline EIF3M & 10480 & eukaryotic translation initiation factor 3 & 1.57 & 1.58 & 1.57 \\
\hline $\mathrm{CD} 247$ & 919 & CD247 molecule & 1.76 & 1.40 & 1.57 \\
\hline CEP27 & 55142 & centrosomal protein $27 \mathrm{kDa}$ & 1.51 & 1.63 & 1.57 \\
\hline RBM34 & 23029 & RNA binding motif protein 34 & 1.56 & 1.55 & 1.56 \\
\hline AKAP11 & 11215 & A kinase (PRKA) anchor protein 11 & 1.62 & 1.49 & 1.55 \\
\hline EXOSC9 & 5393 & exosome component 9 & 1.67 & 1.44 & 1.55 \\
\hline PGRMC2 & 10424 & progesterone receptor membrane component 2 & 1.62 & 1.47 & 1.54 \\
\hline CNBP & 7555 & CCHC-type zinc finger, nucleic acid binding protein & 1.54 & 1.51 & 1.53 \\
\hline SLC4A5 & 57835 & solute carrier family 4 , sodium bicarbonate cotransporter, member 5 & 1.50 & 1.53 & 1.52 \\
\hline PTPLB & 201562 & $\begin{array}{l}\text { protein tyrosine phosphatase-like (proline instead of catalytic } \\
\text { arginine), member b }\end{array}$ & 1.45 & 1.53 & 1.49 \\
\hline TRMT112 & 51504 & tRNA methyltransferase 11-2 homolog (S. cerevisiae) & 1.58 & 1.36 & 1.46 \\
\hline NARS & 4677 & asparaginyl-tRNA synthetase & 1.43 & 1.39 & 1.41 \\
\hline
\end{tabular}

AMI, acute myocardial infarction; UA, unstable angina; ACS, acute coronary syndrome. 


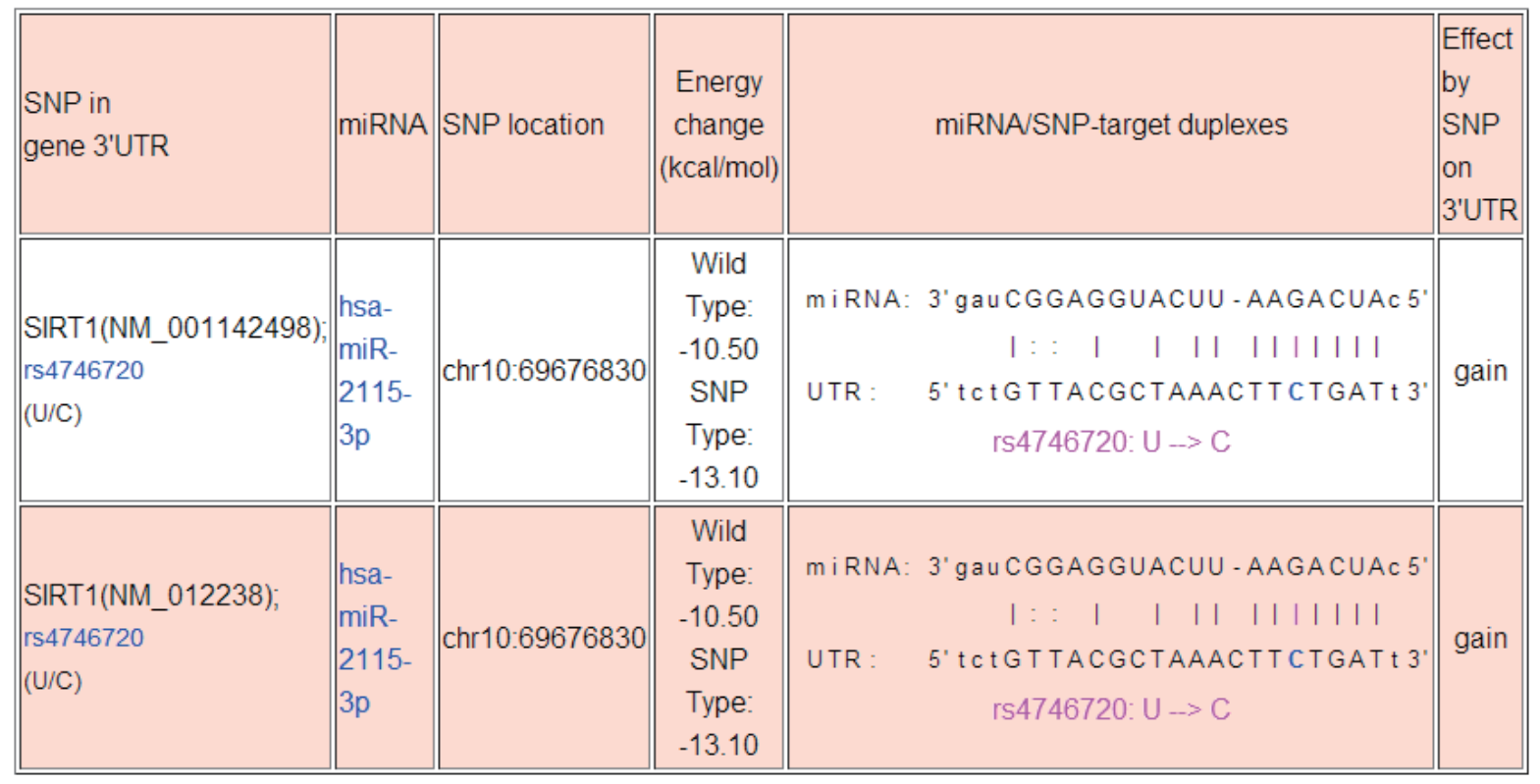

Supplementary Fig. 1.

miRNASNP2 prediction results for rs 4746720

A

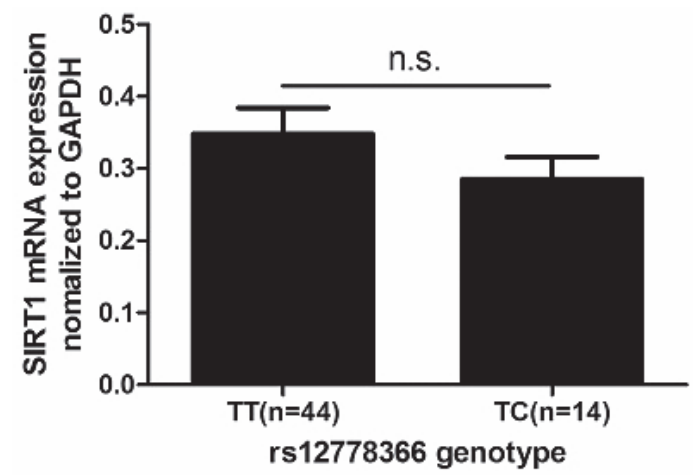

C

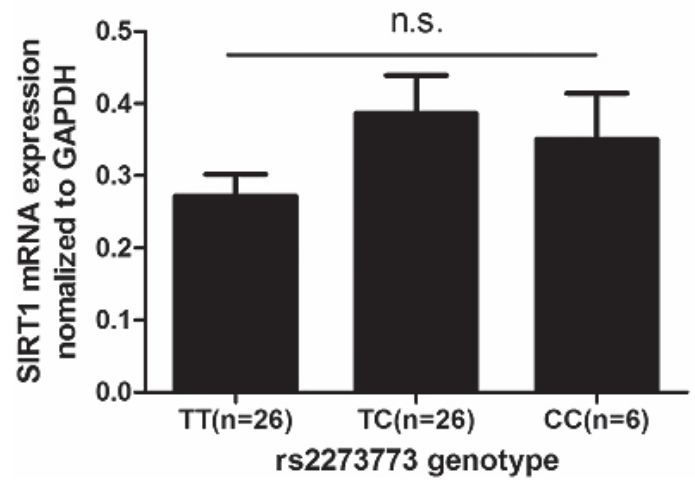

B

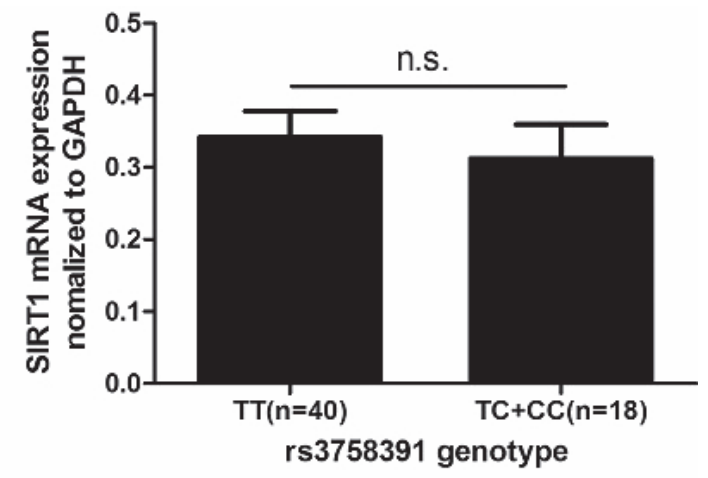

D

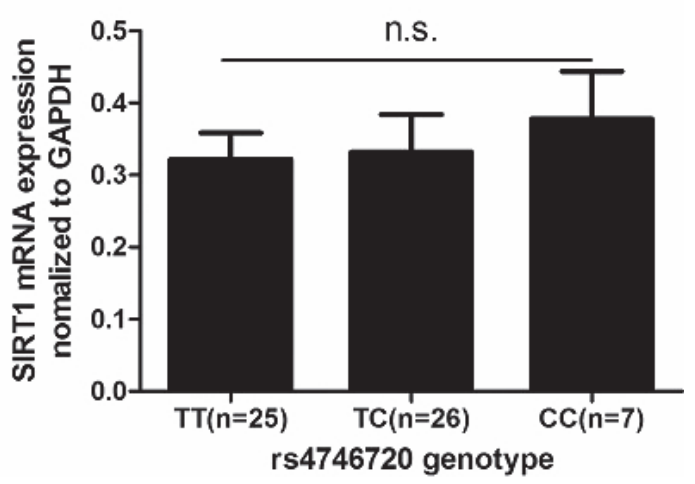

Supplementary Fig. 2 .

Relationships between the SIRT1 mRNA expression levels in the ACS patients and the genotypes of rs12778366 rs3758391, rs2273773 and rs4746720 
Supplementary Table 5.

Characteristics and rs3758391 genotype distributions of the study participants

\begin{tabular}{lccc}
\hline \multirow{2}{*}{ Characteristics } & \multicolumn{2}{c}{ rs3758391 } & p-value \\
\cline { 2 - 3 } & TT $(n=175)$ & TC +CC $(n=54)$ & 0.91 \\
\hline Age & $50.65 \pm 5.67$ & $50.76 \pm 5.73$ & 0.87 \\
BMI $\left(\mathrm{kg} / \mathrm{m}^{2}\right)$ & $25.05 \pm 2.71$ & $24.96 \pm 2.85$ & 0.92 \\
GLU $(\mathrm{mg} / \mathrm{dL})$ & $102.34 \pm 21.90$ & $103.71 \pm 23.99$ & 0.76 \\
CHOL (mg/dL) & $177.92 \pm 47.77$ & $175.35 \pm 45.80$ & 0.12 \\
TG $(\mathrm{mg} / \mathrm{dL})$ & $123.60 \pm 74.84$ & $103.83 \pm 57.09$ & 0.83 \\
SBP $(\mathrm{mmHg})$ & $130.14 \pm 12.85$ & $130.77 \pm 12.74$ & 0.47 \\
DBP $(\mathrm{mmHg})$ & $83.20 \pm 8.22$ & $82.41 \pm 7.86$ & 0.62 \\
Smoker $(\%)$ & 13.7 & 11.1 & 0.27 \\
Drinker $(\%)$ & 28 & 20.4 & \\
\hline
\end{tabular}

*Comparison of the TT genotype with the TC and CC genotypes. Independent Student's $t$-test for continuous variables, Chi-square test for categorical variables. Continuous variables, mean \pm SD. BMI, body mass index; GLU, glucose; CHOL, cholesterol; TG, triglycerides; SBP, systolic blood pressure; DBP, diastolic blood pressure.

\section{Supplementary Table 6.}

Characteristics and rs12778366 genotype distributions of the study participants

\begin{tabular}{lccc}
\hline \multirow{2}{*}{ Characteristics } & \multicolumn{2}{c}{ rs12778366 } & \multirow{2}{*}{-value $^{*}$} \\
\cline { 2 - 3 } & TT $(n=178)$ & TC + CC $(n=48)$ & 0.29 \\
\hline Age & $50.88 \pm 12.41$ & $49.9 \pm 5.34$ & 0.04 \\
BMI $\left(\mathrm{kg} / \mathrm{m}^{2}\right)$ & $24.83 \pm 2.78$ & $25.75 \pm 2.43$ & 0.73 \\
GLU $(\mathrm{mg} / \mathrm{dL})$ & $102.93 \pm 21.56$ & $101.66 \pm 25.87$ & 0.13 \\
CHOL (mg/dL) & $180.11 \pm 46.68$ & $168.49 \pm 44.61$ & 0.37 \\
TG $(\mathrm{mg} / \mathrm{dL})$ & $121.14 \pm 76.16$ & $110.50 \pm 49.58$ & 0.85 \\
SBP $(\mathrm{mmHg})$ & $130.39 \pm 12.41$ & $129.99 \pm 14.02$ & 0.80 \\
DBP $(\mathrm{mmHg})$ & $83.03 \pm 7.71$ & $82.70 \pm 9.32$ & 0.69 \\
Smoker $(\%)$ & 12.4 & 14.6 & 0.35 \\
Drinker $(\%)$ & 27.5 & 20.8 & \\
\hline
\end{tabular}

*Comparison of the TT genotype with the TC and CC genotypes. Independent Student's $t$-test for continuous variables, Chi-square test for categorical variables. Continuous variables, mean \pm SD. BMI, body mass index; GLU, glucose; CHOL, cholesterol; TG, triglycerides; SBP, systolic blood pressure; DBP, diastolic blood pressure. 


\section{Supplementary Table 7.}

Characteristics and rs2273773 genotype distributions of the study participants

\begin{tabular}{|c|c|c|c|c|}
\hline Characteristics & \multicolumn{3}{|c|}{ rs2273773 } & $p$-value ${ }^{*}$ \\
\hline Age & $50.86 \pm 5.79$ & $50.15 \pm 5.38$ & $52.06 \pm 6.49$ & 0.39 \\
\hline BMI $\left(\mathrm{kg} / \mathrm{m}^{2}\right)$ & $25.45 \pm 2.71$ & $24.51 \pm 2.63$ & $24.30 \pm 2.94$ & 0.03 \\
\hline GLU (mg/dL) & $104.54 \pm 23.72$ & $102.82 \pm 20.62$ & $89.80 \pm 17.94$ & 0.04 \\
\hline CHOL (mg/dL) & $176.79 \pm 46.73$ & $177.36 \pm 46.96$ & $180.52 \pm 54.63$ & 0.96 \\
\hline $\mathrm{SBP}(\mathrm{mmHg})$ & $130.56 \pm 13.54$ & $129.00 \pm 12.02$ & $132.84 \pm 10.08$ & 0.45 \\
\hline DBP (mmHg) & $83.54 \pm 8.24$ & $81.48 \pm 8.06$ & $86.55 \pm 10.08$ & 0.03 \\
\hline Smoker (\%) & 11.6 & 14.6 & 17.6 & 0.70 \\
\hline Drinker (\%) & 29.8 & 22.5 & 23.5 & 0.48 \\
\hline
\end{tabular}

*Comparison among the TT, TC and CC genotypes. Analysis of variance (ANOVA) for continuous variables, Chi-square test for categorical variables. Continuous variables, mean \pm SD. BMI, body mass index; GLU, glucose; CHOL, cholesterol; TG, triglycerides; SBP, systolic blood pressure; DBP, diastolic blood pressure.

\section{Supplementary Table 8.}

Characteristics and rs4746720 genotype distributions of the study participants

\begin{tabular}{|c|c|c|c|c|}
\hline Characteristics & \multicolumn{3}{|c|}{ rs 4746720} & $p$-value \\
\hline Age & $50.78 \pm 5.87$ & $50.45 \pm 5.36$ & $51.00 \pm 6.11$ & 0.83 \\
\hline BMI $\left(\mathrm{kg} / \mathrm{m}^{2}\right)$ & $24.40 \pm 2.52$ & $25.43 \pm 2.76$ & $24.95 \pm 2.86$ & 0.06 \\
\hline GLU (mg/dL) & $98.42 \pm 22.42$ & $103.55 \pm 22.71$ & $105.79 \pm 21.26$ & 0.18 \\
\hline CHOL (mg/dL) & $167.99 \pm 44.74$ & $181.15 \pm 50.84$ & $180.18 \pm 41.29$ & 0.19 \\
\hline SBP $(\mathrm{mmHg})$ & $129.33 \pm 12.17$ & $131.52 \pm 12.54$ & $128.88 \pm 14.01$ & 0.36 \\
\hline DBP (mmHg) & $82.17 \pm 8.23$ & $83.48 \pm 8.29$ & $83.06 \pm 7.07$ & 0.59 \\
\hline Smoker (\%) & 15.6 & 11.7 & 13 & 0.76 \\
\hline Drinker (\%) & 18.8 & 25.2 & 37 & 0.08 \\
\hline
\end{tabular}

*Comparison among the TT, TC and CC genotypes. Analysis of variance (ANOVA) for continuous variables, Chi-square test for categorical variables. Continuous variables, mean \pm SD. BMI, body mass index; GLU, glucose; CHOL, cholesterol; TG, triglycerides; SBP, systolic blood pressure; DBP, diastolic blood pressure.

Supplementary Table 9.

Linkage disequilibrium plot of the SNPs with $r^{2}$ among the 229 healthy subjects

\begin{tabular}{|c|c|c|c|c|}
\hline$r^{2}$ & rs12778366 & rs3758391 & rs2273773 & rs 4746720 \\
\hline rs 12778366 & & 0.02 & 0.13 & 0.05 \\
\hline rs3758391 & & & 0.12 & 0.05 \\
\hline rs 2273773 & & & & 0.33 \\
\hline rs4746720 & & & & \\
\hline
\end{tabular}

